\title{
Metastatic Malignant Neoplasm in the Urethra
}

National Cancer Institute

\section{Source}

National Cancer Institute. Metastatic Malignant Neoplasm in the Urethra. NCI

Thesaurus. Code C7573.

A malignant neoplasm that has spread to the urethra from another anatomic site. 